\title{
IMPLEMENTATION OF ARTIFICIAL NEURAL NETWORK DATA MINING ALGORITHM: A CASE STUDY OF BIRTH REGISTRATION DATA
}

\author{
Dr. P. Y. Desai \\ Associate Professor, Department of Information and Communication Technology, \\ Veer Narmad South Gujarat University, Gujarat, India
}

\begin{abstract}
The scope of different applications of Artificial Neural Network is immense. In recent times, Artificial Neural Network has been used in different type of applications. The use of Artificial Neural Network in Medical Science, Financial Markets, Employee Hiring, Fraud Detection, Cyber security are well know. In this paper, the use of Artificial Neural Network is proposed for Birth Registration e-governance data. The practical implementation was done using Microsoft Analysis Service and results indicates that interesting relationship can be obtained using Microsoft Neural Network data mining algorithm.
\end{abstract}

Key words: E-Governance, Artificial Neural Network, Data Mining.

Cite this Article: Dr. P.Y. Desai, Implementation of Artificial Neural Network Data Mining Algorithm: A Case Study of Birth Registration Data. International Journal of Computer Engineering and Technology, 10(1), 2019, pp. 48-53.

http://iaeme.com/Home/issue/IJCET?Volume=10\&Issue=1

\section{INTRODUCTION}

The Microsoft Neural Network algorithm can be utilized to create artificial neural paths. These paths are used as patterns and these patterns can be used for future predictions. The Microsoft Neural Network algorithm can be effectively utilized for detecting very complex relationship among many input and output attributes [1]. Microsoft Neural Network algorithm can span up to two levels. In the single-level, inputs are connected directly to the outputs whereas, in the two-level case, input combinations effectively become new inputs, which are then connected to the outputs. The level that transforms certain input combinations into new inputs is referred to as a hidden layer [1]. Considering the functioning of Microsoft Neural Network algorithm, various attributes of Birth Registration data are utilized as input and output attributes. 


\section{RESEARCH METHODOLOGY}

\subsection{Configuration of Model}

In this Microsoft Neural Network Model, "Birth Date" attribute was kept as Key field as it containing unique value for each row of the dataset. The input attributes were Father Education ID, Mother Education ID and Religion ID. The output attributes were Delivery Attention ID and Delivery Method ID. The setting of various attributes is shown in the Figure 1.

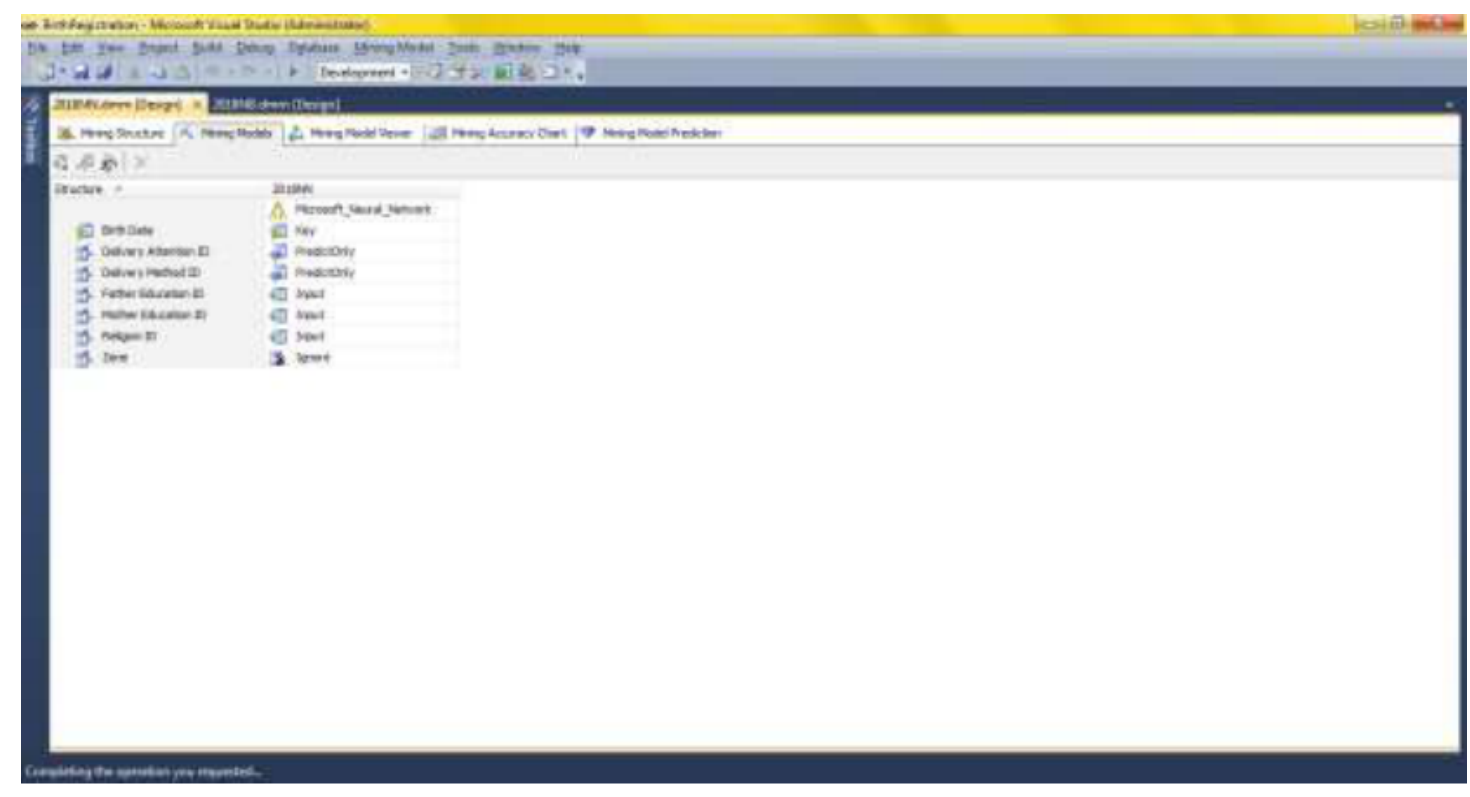

Figure 1 Setting of input and output attribute in ANN

The input attributes form the first layer of the Microsoft Neural Network. Here, each input attribute is mapped to one node [1]. The Microsoft Neural Network algorithm converts input attribute to a floating point number in the same scale, which is typically between -1 to 1 [1]. The hidden nodes in the hidden layer get input either from the input layer or previous hidden layer. The hidden nodes combines all the input based on the weight of associated edges, process calculation and produce value which is provided to subsequent layer [1]. The output nodes is predictable attribute, which is Delivery Attention ID and Delivery Method ID in this mining model.

\subsection{The Combination and Activation Functions}

In Neural Network, a Neuron has number inputs and one output. A Neuron combine all the input values which is referred was combination, does some calculation and generate some output value. This is referred as activation function [1]. The Microsoft Neural Network algorithm uses weighted sum approach and then output of the combination is passed on to activation function [1]. Microsoft Neural Network uses tanh as the activation function in the hidden nodes and sigmoid activation function in the output nodes [1].

$$
\begin{aligned}
& \text { sigmoid: } 0=1 /\left(1+e^{a}\right) \\
& \text { tanh: } 0=\left(e^{a}-e^{-a}\right) /\left(e^{a}+e^{-a}\right)
\end{aligned}
$$




\subsection{Processing of the Model}

Considering input and output attributes, combination function and activation function the Microsoft Neural Network algorithm processes model using backpropagation [1]. The main steps are as follows [1]:

Step1: Randomly assign value to all weights in the neural network

Step2: Calculate output based on the current weight for training data

Step 3: Calculate output error, the backpropagation method calculate error for each output and hidden neuron. The weights are recalculated.

Step 4 Step 2 is repeated till condition is satisfied.

Considering 2.1, 2.2 and 2.3, The Microsoft Neural Network was generated for Birth Registration Data.

\section{INTERPRETING THE MODEL AND RESULTS}

The Microsoft Neural Network algorithm does not display graphical display of the trained neural network's layout. The Microsoft Neural Network viewer shows the impact of attribute/value (AV) pairs related to the predictable attribute. It evaluates the impact of each AV related to the predictable states and then sorts based on the following score

$$
\text { Score }=\mathrm{P}(\mathrm{AV} \mid \text { Predictable State } 1) / \mathrm{P}(\mathrm{AV} \mid \text { Predictable State } 2)
$$

The Neural Network algorithm can calculate P( Predictable_State | AV). The method used to get this probability is to set all the other input attributes as Missing and the AV as the only input neuron. The neural network then calculates the probability of a predictable attribute state [1]. Because you have the marginal states of $\mathrm{P}$ (Predictable State 1), P(Predictable State $2)$, and $\mathrm{P}(\mathrm{AV})$, you can derive $\mathrm{P}(\mathrm{AV} \mid$ Predictable State 1$)$ using the Bayes rule as follows:

$\mathrm{P}(\mathrm{AV} \mid$ Predictable State 1$)=$

$\mathrm{P}($ Predictable State $1 \mid \mathrm{AV}) * \mathrm{P}(\mathrm{AV}) / \mathrm{P}($ Predictable State 1)

The different states of Religion ID, Father Education ID and Mother Education ID attributes are shown in the Figure 2 and Figure 3 respectively.

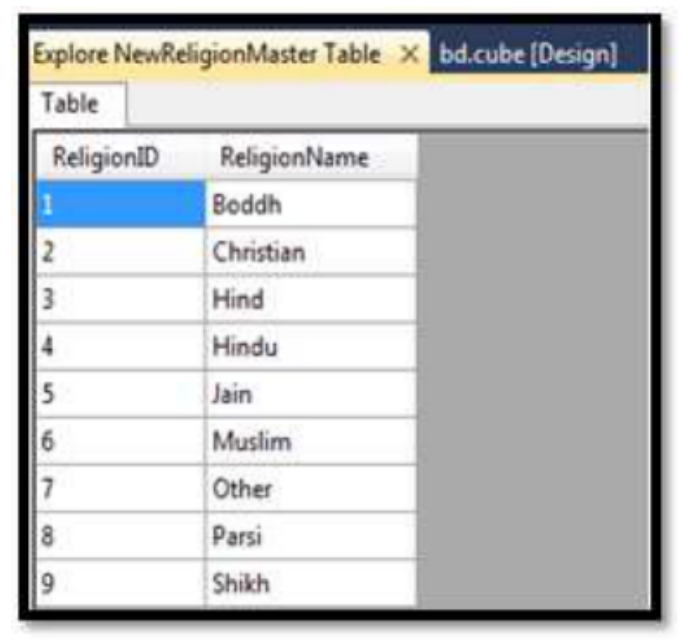

Figure 2 Religion ID attributes's states 


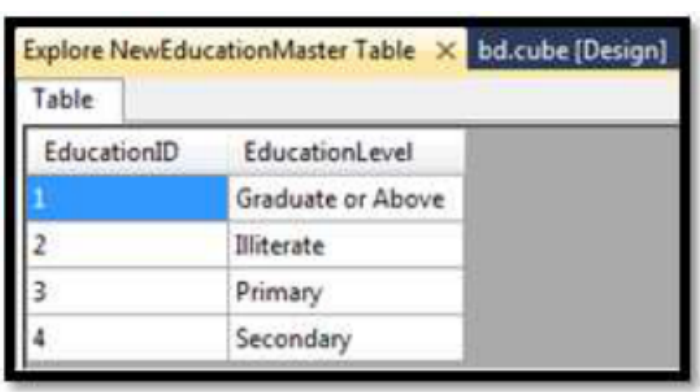

Figure 3 Father Education ID and Mother Education ID attributes' states

The Medical Attention Type predictable attribute, the Microsoft Neural Network generates impact of Attribute Value pair as shown in the Figure 5.

Explore DeleiveryMethodName Table $\times$ bd.cube [D
\begin{tabular}{|l|l|}
\hline Table & \\
\hline DeleiveryMethodID & DeliveryMethodName \\
\hline 1 & Caesarean \\
\hline 2 & Forceps/Vaccum \\
\hline 3 & Natural \\
\hline
\end{tabular}

Figure 4 Delivery Method ID attributes' states

The result clearly indicates that Religion ID $=1$, which is Boddh religion has highest impact for Delivery Method ID=2, which is Forceps/ Vaccum.

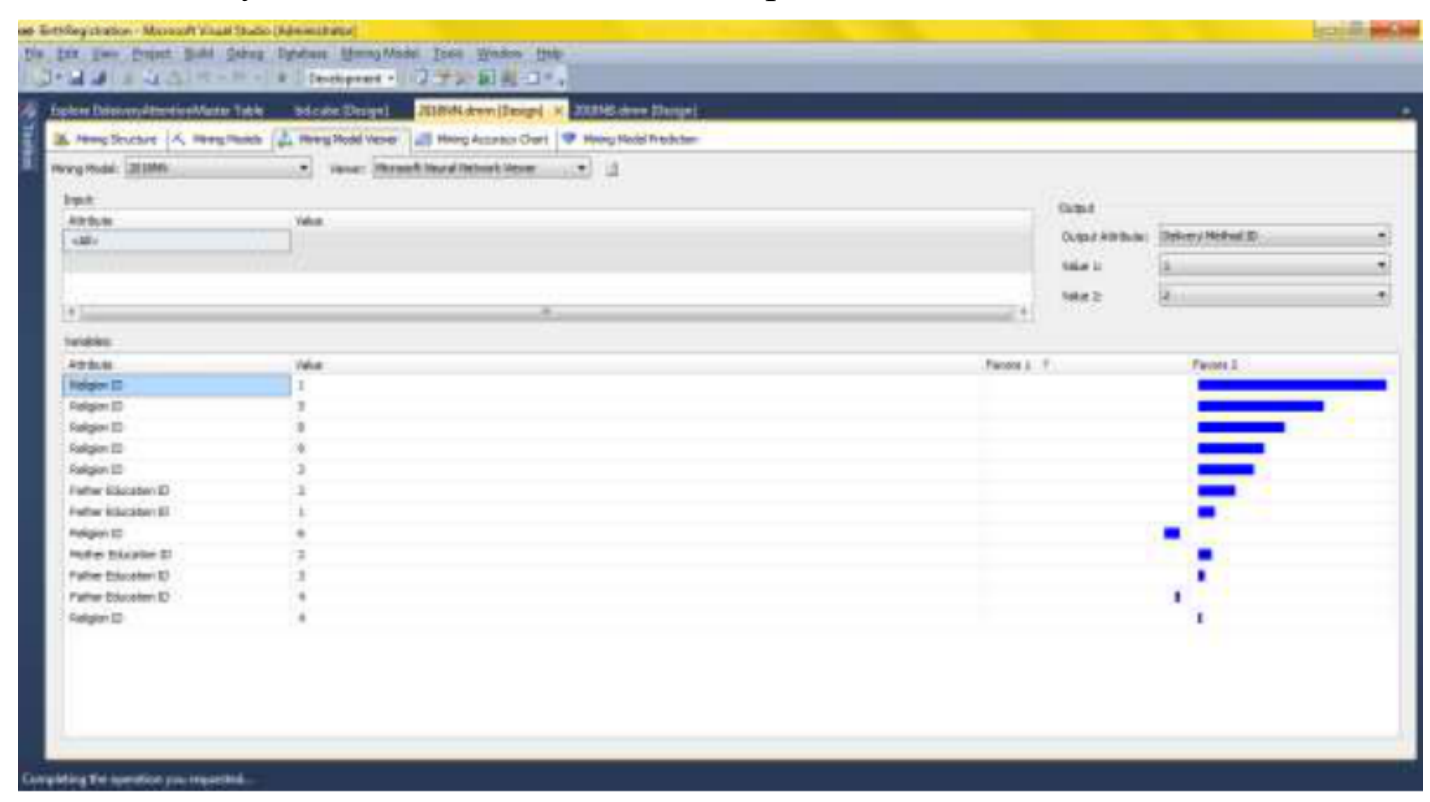

Figure 5 Impact Analysis for Delivery Method ID attribute (ID=1 and ID=2)

Similarly, Religion ID $=5$, which is Jain Religion has highest impact for Delivery Method $\mathrm{ID}=1$, which is Caesarean. The result is shown in the Figure 6. 
Implementation of Artificial Neural Network Data Mining Algorithm: A Case Study of Birth Registration Data

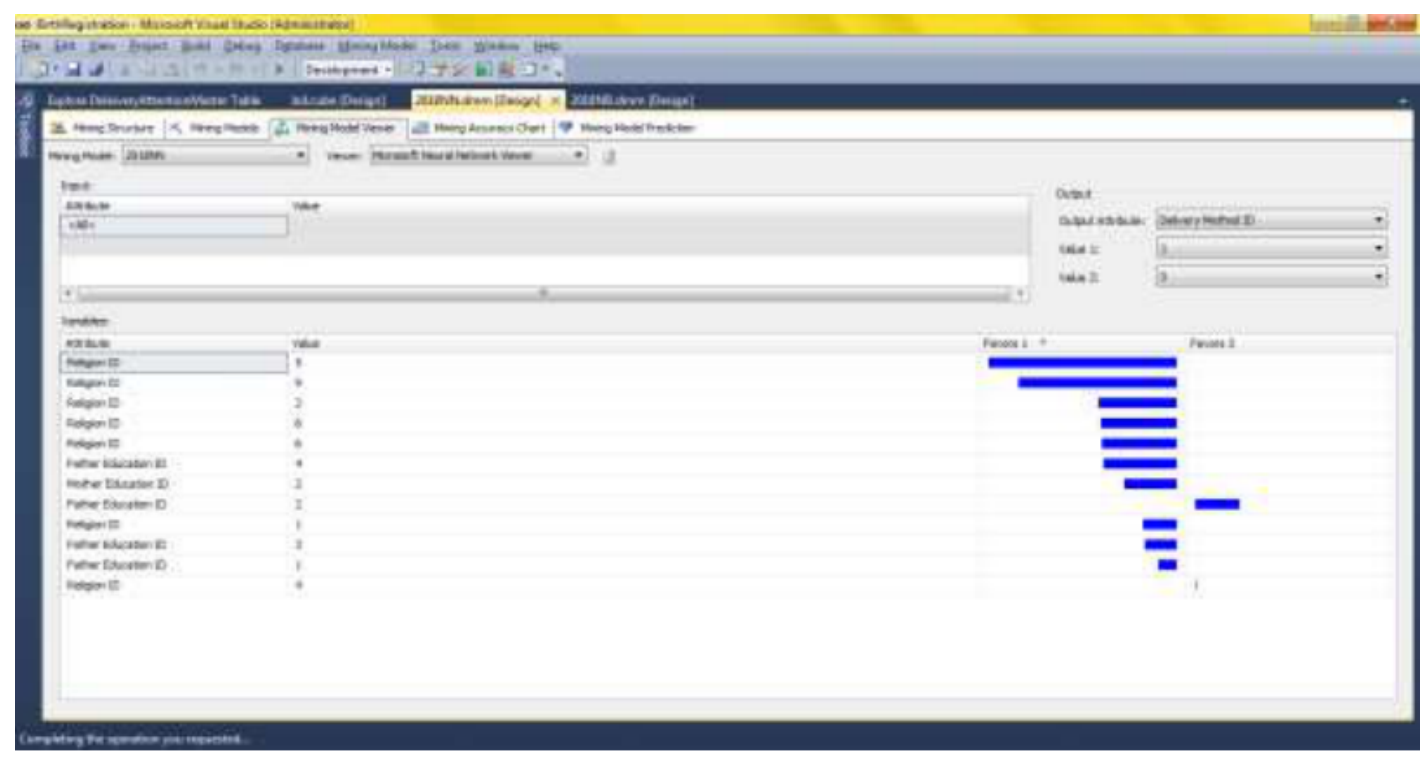

Figure 6 Impact Analysis for Delivery Method ID attribute (ID=1 and ID=3)

For Medical Attention ID, various states are shown in the Figure 7.

Explore DeleiveryAttentionMaster Table $\times$ bd.cube [Design]
\begin{tabular}{|l|l|}
\hline Table & \\
\hline DelieveryAttentionID & DelieveryAttentionName \\
\hline 1 & Doctor, Nurse or Trained Midwife \\
\hline 2 & Institutional-Government \\
\hline 3 & Institutional-Private or Non-Government \\
\hline 4 & Relatives or Other \\
\hline 5 & Traditional Birth Attendant \\
\hline
\end{tabular}

Figure 7 Delivery Attention ID attributes' states

The result shows that Religion ID $=6$, which is Muslim religion has highest impact for Delivery Attention ID=2, which is Institutional-Government. The result is shown in the Figure 8.

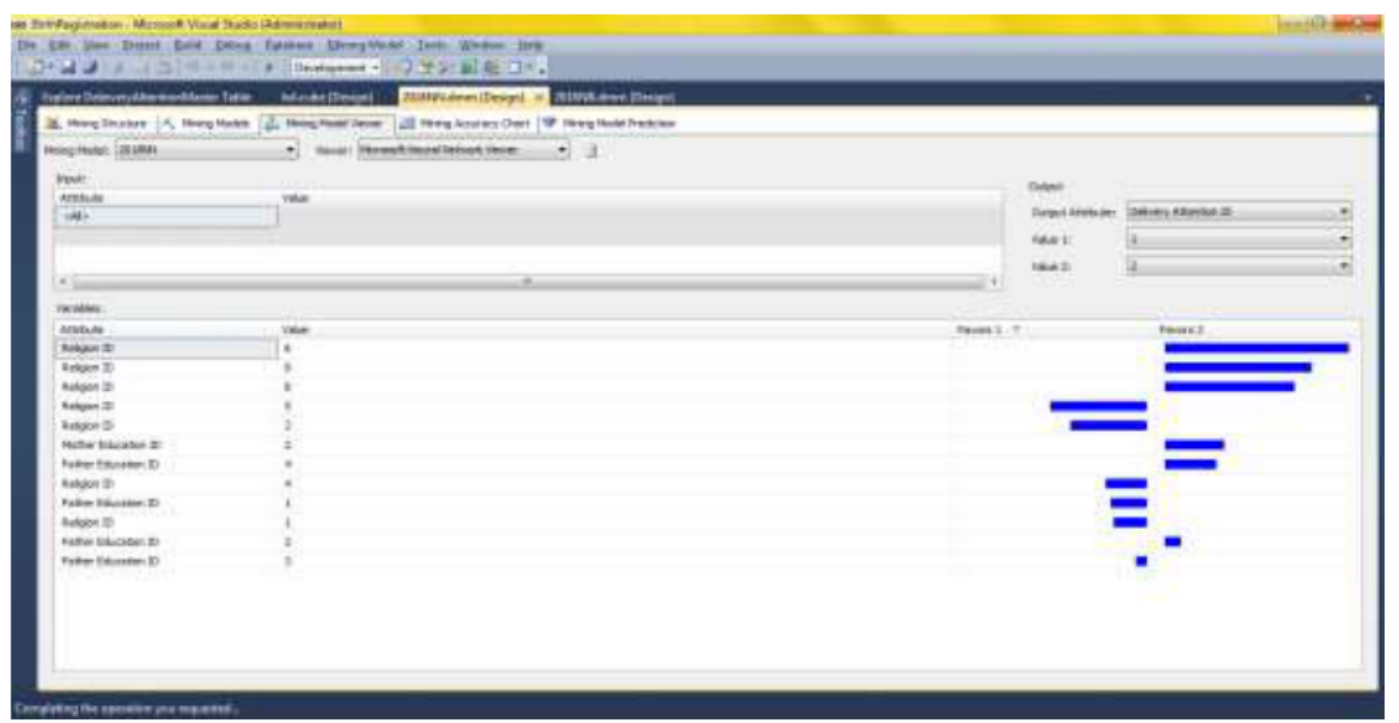

Figure 8 Impact Analysis for Delivery Attention ID attribute (ID=1 and ID=2) 
In another impact analysis, the result shows that Religion ID $=8$, which is Parsi religion has highest impact for Delivery Attention ID=1, which is Doctor, Nurse, Trained Midwife. The result is shown in the Figure 9.

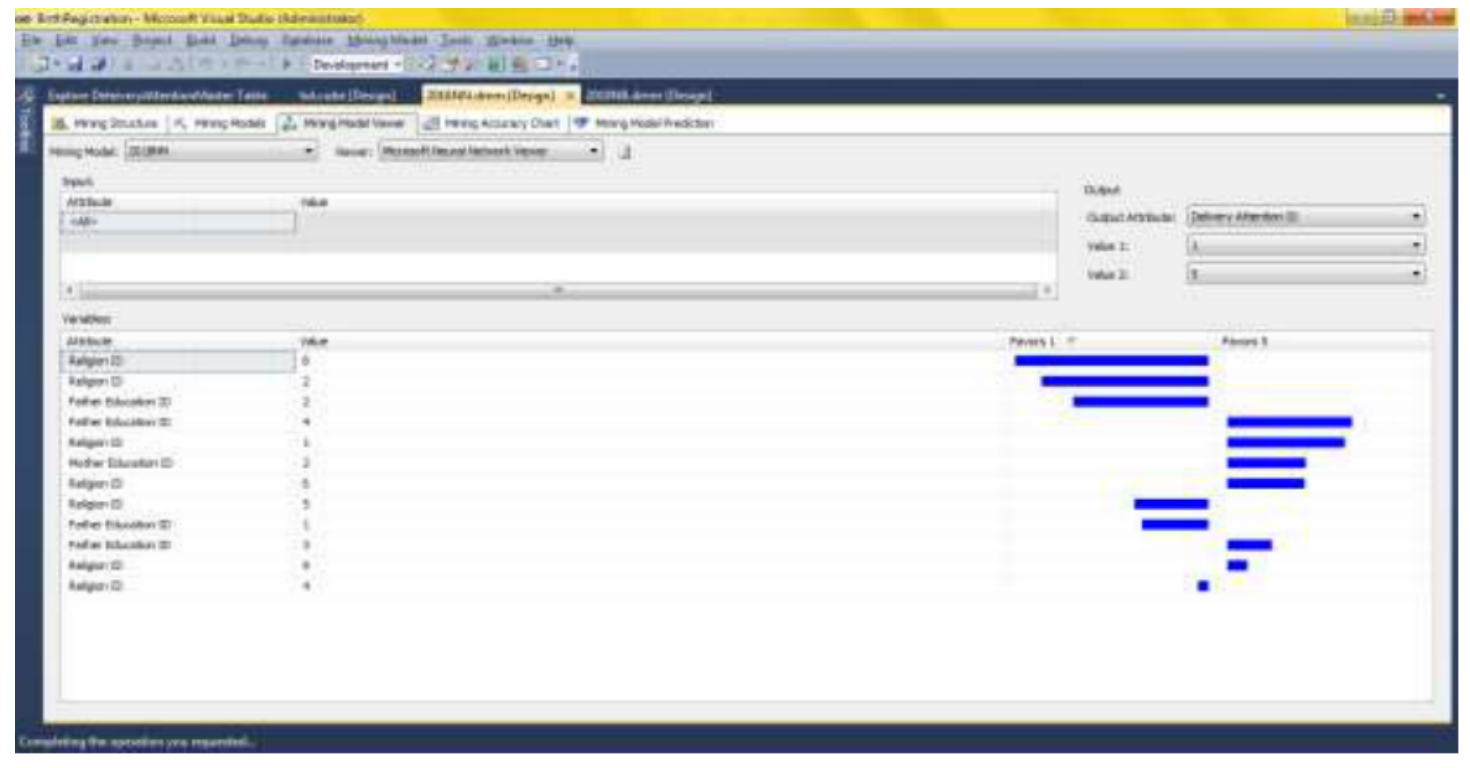

Figure 9 Impact Analysis for Delivery Attention ID attribute (ID=1 and ID=5)

\section{CONCLUSIONS}

The analysis of Microsoft Neural Network for Birth Registration data shows that new and interesting relationships can be derived. The results confirm that Neural Network should be adopted for better understanding of e-governance data.

\section{REFERENCES}

[1] Jamie et al, Data Mining with Microsoft SQL Server 2008.

[2] Microsoft Neural Network Algorithm, Last Access Date: 4 Feb 19 https://docs.microsoft.com/en-us/sql/analysis-services/data-mining/microsoft-neuralnetwork-algorithm?view=sql-server-2017

[3] Microsoft Neural Network Algorithm Technical Reference https://docs.microsoft.com/enus/sql/analysis-services/data-mining/microsoft-neural-network-algorithm-technicalreference?view=sql-server-2017

[4] Mining Model Content for Neural Network Models (Analysis Services - Data Mining) https://docs.microsoft.com/en-us/sq1/analysis-services/data-mining/mining-model-contentfor-neural-network-models-analysis-services-data-mining?view=sql-server-2017

[5] Sujata S.Kharat and Vamshi Krishna, To Study Artificial Neural Networks in Data Mining and Its Method, International Journal of Advance Research in Computer Science and Management Studies, Volume 3, Issue 7, July 2015.

[6] Bhushan C. Kamble, Speech Recognition Using Artificial Neural Network - A Review, Int'l Journal of Computing, Communications \& Instrumentation Engineering. (IJCCIE) Vol. 3, Issue 1, 2016.

[7] Zahed Soltani and Ahmad Jafarian, A New Artificial Neural Networks Approach for Diagnosing Diabetes Disease Type II, (IJACSA) International Journal of Advanced Computer Science and Applications, Vol. 7, No. 6, 2016. 